\title{
La inserción a la docencia: una política pendiente en el marco de un sistema de formación permanente en Honduras
}

\author{
Dora Suyapa Díaz Quinteros \\ Doctora en Gestión del Desarrollo Humano en Ciencias Sociales \\ Docente e investigadora en temáticas socio educativas \\ Universidad Nacional Autónoma de Honduras, Honduras \\ dora.diaz@unah.edu.hn
}

Recepción: 03-04-2018 / Aceptación: 22-06-2018

\section{Resumen}

El presente artículo tiene como propósito analizar los resultados de la investigación realizada el año 2017, a docentes del sistema educativo hondureño en los niveles de Básica y Media en zona urbana y rural, con el interés de plantear un aporte científico que contribuya a fortalecer un programa de formación docente novel como sistema a la inserción.

El estudio hace énfasis en cuatro aspectos: la inserción del docente novel, programa integral, formación permanente y la identidad del docente. La información empírica fue obtenida por docentes activos en los departamentos de Francisco Morazán, Cortes, Atlántida, Choluteca, Santa Bárbara, ubicados en la zona central, sur, occidente de Honduras. En el trabajo de campo participaron asistentes técnicos de las Direcciones Departamentales correspondientes.

Al realizar el estudio se detectó que el sistema educativo hondureño está pendiente de implementar un programa de inserción a docente novel, intermedio y experimentado; siendo una realidad educativa para ser discutido en la agenda de las autoridades educativas y tomar la decisión. Asimismo, de ser posible, construir una política educativa en forma acertada, participativa y con actores clave. Esta realidad de no contar con programas de inserción refleja debilidad del ejercicio de ser docente y, consecuentemente, en las competencias dentro del ejercicio de la docencia; también, debilidad en el concepto sobre la identidad y los procedimientos de contratación docente. Todo esto se estaría reflejando en los indicadores de la calidad educativa.

En este sentido, el estudio utilizó como instrumento metodológico técnicas cualitativas como las de grupos colaborativos y entrevistas, las cuales contienen una serie de datos sobre la caracterización de programas de inserción, la identidad del ejercicio docente, los procedimientos de contratación; así como la caracterización de los modelos de capacitación implementados. Además, se utilizó el método de teoría fundamentada de referentes teóricos y documentos oficiales.

Palabras clave: Política de inserción, docente novel, programa integral, formación permanente.

\begin{abstract}
The purpose of this article is to analyze the results of a research carried out in 2017, based on the Honduran Education System in the elementary and high school levels in urban and rural areas in order to create a scientific proposal that contributes to strengthen a teacher-training program as a system of insertion.

The study emphasizes four aspects: the insertion of the inexperienced teachers, integral program, permanent education, and the identity of teachers. The empirical information was obtained by active teachers in the departments of Francisco Morazán, Cortes, Atlántida, Choluteca, and Santa Bárbara, located in the central, south, and west zone of Honduras. Technical assistants of the corresponding departmental directors participated in the fieldwork.

When carrying out the research, it was detected that the Honduran Educational System is pending to implement a program of insertion to inexperienced, intermediate, and experienced teachers; this is an educational reality to be discussed in the agenda of educational authorities and make proper decisions. Likewise, if possible, it should be created an educational policy in an accurate, participatory way with key actors. As this reality does not have integrated programs, it reflects weaknesses in the exercise of being a teacher and, consequently, in the competences within the teaching profession itself. It also presents weaknesses in the concept of identity and teacher recruitment procedures. All this would be reflected in the indicators of educational quality.

In this way, the study used methodological tools and qualitative techniques such as, collaborative groups and interviews, which contained a series of data on the characterization of insertion programs, the identity of the teaching exercise, the hiring procedures as well as the characterization of implemented training models. Additionally, it was used a method grounded in theoretical references and official documents.
\end{abstract}

Keywords: Insertion policy, unexperienced teacher, integral program, permanent education. 


\section{Introducción}

La investigación realizada enmarca dos categorías: la inserción y el sistema de formación permanente o desarrollo profesional -como lo llaman otros teóricos- se propone aportar líneas a programas de inserción docente en el sistema educativo hondureño, específicamente en los niveles de básica y media, cuya finalidad es generar debate científico y aportar metodológicamente líneas sobre la relación vinculante entre los subsistemas de formación inicial y permanente. Para profundizar esta relación ha sido necesario y de interés investigativo, indagar sobre los sistemas de formación del profesorado y sus tendencias pedagógicas en otras experiencias de países en la región, las cuales confluyen en diferentes formas de insertar al docente novel o principiante al quehacer educativo, a través de programas de inserción. Asimismo, el estudio indaga específicamente temas relacionados a las formas de contratación a docentes y los enfoques de capacitación, estableciendo valoraciones a la etapa de inserción específicamente un programa sistémico del docente novel, intermedio y experimentado; una reflexión pedagógica cuyo propósito es incidir en la política educativa.

Generar una política educativa que atienda a través de un programa estructurado que esté relacionado a certificar y acreditar la formación docente integral, ha sido fundamental la revisión teórica y verificación empírica para sustentar el aporte científico para la creación de una política de Estado, que contribuya desde esta categoría. En este sentido, la iniciativa es un aporte metodológico desde la mirada de informantes actores, que apuntan este vacío y plantean que deberían tener acceso. Está afirmación se confirmó en el andamiaje de la investigación; es decir, en la forma del cómo se insertan al ejercicio de ser docente; consecuentemente, agrava la situación al no contar con este tipo de programas de formación $y$, por ende, capacitaciones aisladas.

Es necesario un enfoque alineado de capacitación estratificado a docentes principiantes, intermedios y experimentados en años de servicio; también la valoración del perfil de ingreso al ejercicio laboral, de esta forma se aseguraría las competencias propias disciplinarias, así como ampliar los niveles de identificación con la carrera docente. Se aspiraría un ejercicio comprometido, innovador, autónomo y comprometido con la transformación de la práctica educativa y la calidad de la misma.

Contar con este tipo de programas, es indudablemente que los procesos formativos fortalecen el perfil del ejercicio docente; asimismo, es incuestionable que el conocimiento es reconocido hoy en día como el recurso estratégico para potenciar la capacidad competitiva de las organizaciones. Sin embargo, cuando se trata de indagar la temática de la formación docente en el contexto de los sistemas educativos, se observa la existencia de 
problemáticas diversas según los contextos del quehacer formativo, lo que hace complejo su estudio y necesario el abordaje de esa realidad con detalle.

Mientras en los países desarrollados los sistemas educativos han mostrado interés creciente por la capacitación y el desarrollo de modelo propio para un sistema de formación integral, como vía para incrementar su capacidad innovadora y la creación de competencias en los estudiantes y en el mismo docente, el abordaje que se ha dado hasta ahora al tema ha sido la adopción de programas de inserción al docente novel o principiante, con enfoque de capacitación que conllevan a fortalecer la identidad del ejercicio del docente.

Al respecto, distintas investigaciones realizadas en sistemas de educación en América Latina y Centro América, orientadas a indagar sobre la importancia que tiene la UNESCO en la formulación de políticas de transformación educativas como las necesidades particulares de cada sistema educativo, acorde con las características de los entornos nacional y mundial, han permitido identificar que existen vacíos sobre el tema de parte de diversos académicos e investigadores en educación, organizaciones y la necesidad de conocimiento más detallado y riguroso sobre la situación actual es este campo; (Vaillant, D., 2007; Salgado, R., 2016; Avalos, B., 2016; Imbermon F., 2009).

Uno de los más importantes debates que contempla esta investigación está relacionado con la búsqueda con un nuevo perfil del docente para el ejercicio en los primeros años desde un programa de formación. Al respecto hoy día se plantea la necesidad de revalorizar las competencias que debe dominar un docente, entre lo que se quiere hacer y lo que termina resultando, planteándose reformas de carácter nacional como local. El estudio recupera un debate sobre el nuevo rol que desempeñan los docentes con jóvenes y niños en las aulas, como un derecho alienable en una educación de calidad desde la formación docente.

A partir de estos debates es particularmente problemático y preocupante porque genera a los tomadores de decisiones a implementar nuevos esquemas de evaluación del desempeño docente, sus incentivos y -probablemente- no así definir un enfoque y concepción del sistema de formación. No obstante, las temáticas están siendo parte de los debates temáticos de las agendas de discusión por parte de las agencias internacionales. Sin embargo, generalmente se valora al docente como un insumo más de la calidad de la educación, junto al texto escolar, el tiempo de instrucción o la biblioteca, entre otros; siendo uno de los más significativos debates en los últimos tiempos.

Uno de los mecanismos más conocidos para identificar estos cuestionamientos está relacionado con las evaluaciones de los sistemas educacionales en América Latina, especialmente en lo referente a proyectos de mejoramiento de la calidad de la educación y las evaluaciones de las reformas educativas. 
La formación está en debate con expertos, académicos, profesores y no solo al ámbito escolar, sino empresarial (formación en la empresa), social (formación para el uso del tiempo libre), político (formación para decidir), etc. Esto significa que cada sistema educativo debió o deberá contar con un sistemade formación docente articulado ¿Por qué?, porque es una necesidad investigar para tomar decisiones, identificando características pertinentes que deben desarrollarse en el sistema en mención; enfocar el análisis de sistemas de formación es también analizar lo que hoy en día se reconoce sobre los estándares de Desempeño Profesional Docente. De ellos se señala establecer características y prácticas de un docente de calidad, quien -además de tener dominio del área que enseña- debe presentar evidencias y peculiaridades que fortalezcan su desempeño; tales como el uso de pedagogía variada, la actualización permanente, la buena relación con los alumnos y padres de familia, una sólida ética profesional, entre otras.

Estos estándares se refieren a todos estos elementos y permiten al docente enmarcar su desempeño dentro de parámetros claros, con el propósito de que los estándares de desempeño profesional docente fomenten en el aula la enseñanza, permitiendo lo óptimo: "todos los estudiantes alcancen perfiles de egreso o aprendizajes declarados por el Currículo Nacional para la Educación Básica y para el Bachillerato". Sin embargo, no se menciona en qué estructura, en qué sistema de formación y si estos están articulados. No obstante, no todos los sistemas educativos favorecen medir por estándares. Para evaluar un sistema educativo y, más cuando se argumenta que la articulación debe ser con un enfoque integral - sistémico, crear un sistema de formación desde las dimensiones como ser, la génesis, la naturaleza, la dinámica e impacto sobre el objeto de estudio, en este caso de la formación, es complejo. Pero síse puede ver qué experiencias de éxito han tenido los países en América Latina e indagar literatura de países europeos.

Focalizar la mirada a la articulación de los sistemas de formación docente, significa identificar y profundizar en forma exhaustiva la participación de las instituciones formadoras; y que esta esté en la agenda tanto política, técnica y académica en los marcos legales y administrativos de la formación docente. Esto con el propósito de desarrollar algunos temas que converjan en el quehacer de formación, es decir en aspectos encontrados en estudios realizados que, de alguna manera, delimitan el campo de análisis; y a la vez el actuar como factores intervinientes en el momento de describir y caracterizar la carrera de docentes. También problemáticas comunes que se dan en los sistemas de formación de ambos, así como en las instituciones formadoras inicial y permanente. 
De modo que cabe subrayar que la mirada en clave articulada conduce a hallar escenarios, actores, problemas y estrategias similares; aunque cada subsistema debe tener especificidades configurando los procesos de toma decisiones. Según el documento, "Claves para una nueva formación del profesorado" del investigador español y experto en esta temática de formación permanente, Imbernón (2001), en los últimos cuarenta años se han construido lineamientos para “adaptar” la formación docente a diferentes momentos históricos en los que los procesos económicos, sociales y tecnológicos se han ido transformando.

Los resultados de la investigación muestran que no se trata solamente de diseñar un sistema de formación, sino de seleccionar el perfil idóneo, acompañado de capacitación por estratos; aprovechar el amplio desarrollo de los recursos tecnológicos disponibles hoy en día, plantear la necesidad que el profesorado se involucre en los procesos de desarrollo profesional $y$, consecuentemente, que contribuya al desarrollo de los contextos escolares y entornos de la misma educación como área de desarrollo humano (Marcelo, 2011). Por otro lado, se debe retomar la "perspectiva de enseñanza - aprendizaje" a lo largo de la vida para los profesores, que implique a los países una atención más destacada, ofrecer apoyo a los profesores en sus primeros años de enseñanza y proporcionar incentivos, recursos para su desarrollo profesional continuo.

\section{Metodología}

La exploración de la realidad de país, en cuanto al sistema actual de formación y capacitación docente, permite identificar las bases conceptuales explicativas del fenómeno que se investiga; conocer la situación actual del contexto hondureño y las expresiones del problema que se investiga para proponer salidas viables con miras a la mejora continua de la educación en los niveles de media y básica en Honduras.

El abordaje metodológico utilizado se basó en el enfoque cualitativo, interpretativo-fenomenológico; seleccionando informantes vinculados a la docencia en servicio activo, en los niveles de Básica y Media ubicados en diferentes departamentos. Se seleccionaron bajo los siguientes criterios: plaza permanente en sector público, años de servicio, ubicación rural y urbana; al final se consultaron 204 personas con criterios de selección y la forma de muestreo intencionado, aplicando entrevistas semi estructuradas, grupos colaborativos y cuestionarios. Para el análisis e interpretación de la información se desarroló un análisis de contenido. 


\section{Resultados}

Para analizar los resultados, la información recopilada se expresa mediante las diferentes categorías que involucró la investigación:

\section{a. Autonomía docente}

\section{Unidad teórica}

\section{Unidad empírica}

...En el informe de Chile sobre Formación El mayor cuerpo de contenido posee un reDocente en América Latina (2011), describe la inserción docente de cada país, menciona el caso de Finlandia, todos los profesores finlandeses se forman actualmente al menos al nivel del grado de Magister y no pocos al nivel de Doctor. Tal grado asegura una preparación académica exigente que claramente garantiza un nivel que permitirá a posteriori la adquisición autónoma de otros conocimientos y una estatura para gistro que la autonomía para la toma decisiones se relaciona altamente en el desarrollo de clases... pues tengo la oportunidad de implementar técnicas y estrategias que considero ayudarán al alumnado...Se resuelven las dificultades en la labor docente. No obstante, la resolución de problemas en esta línea escasamente mencionan la integración en docentes para situaciones académicas en relacionarse con el saber en desarrollo, la investigación, las otras disciplinas y sus cultores, que permite la adquisición crítica de nuevas ideas y la incorporación pertinente de innovaciones.

\section{Unidad de significado}

El recuento de frecuencia se genera en la relación de la autonomía con el quehacer educativo de aula, es decir en el momento de establecer acomodación de estrategias de enseñanza y aprendizaje, no se considera la autonomía como concepto de aseguramiento académico y tampoco que emane redes de docentes para registrar la autonomía educativa como lo requiere la implementación de adecuaciones curriculares. Por lo tanto, el sistema escolar sigue estando altamente centralizado.

Figura 1. Autonomía en relación a los años de servicio para la toma de decisiones en el quehacer educativo. 


\section{b. Definición actual de docente}

Unidad teórica

...La inserción docente desde la construcción de su perfil identaria; según Vaillant (2007)," la construcción identitaria docente comienza con la formación inicial, pero de igual forma, la autora advierte que la identidad se fortalece o desarrolla durante el desempeño de la profesión, un aspecto interesante, desde la postura de la identidad como proceso de construcción subjetiva es que ésta no se acredita con un título".

Navarrete (2017), "Por ello se debe estudiar la identidad desde los contextos de práctica. De acuerdo con el interés de este estudio se prestará atención a los procesos de tránsito hacia la identidad durante el ejercicio de la función, de un profesorado que no contó con la oportunidad de formarse en cuestiones referidas a la docencia antes de iniciarse como tal."

Venegas (2009) plantea que “... la imagen profesional del docente tiene, en la calidad de la formación del formador, [...] su mayor fortaleza o su peor tragedia”. Este es un pensamiento sugerente, y a la vez inquisitivo, que nos llama a colocar la atención en un tema de importancia notable: ¿quiénes, dentro de la sociedad, se hacen cargo de la preparar a los nuevos docentes? Este aspecto es poco investigado y documentado en Centroamérica y República Dominicana.

\section{Unidad empírica}

...ya no tenemos identidad porque todos nuestros derechos son violentados; el ser docente en el sistema hoy en día es bien hostigaste; nuestra identidad ha quedado desvaluada por el irrespeto nuestros derechos. No obstante en su minoría versan que es un ente pulcro, un personaje clave, como una carrera digna, en donde se prepara el recurso humano para el desarrollo de la nación; ...es una actividad laboral digna; ...bueno la definiría que como docente no contamos con el apoyo en las aulas de clase falta de libro, mobiliario...

\section{Unidad de significado}

Las unidades emergentes de la definición de identidad se conceptualizan en su mayoría en una profesión desvalorizada en relación a la violación de los derechos, el cual ha decrecido un verdadero apostolado, con la vocación magisterial; surgiendo una identidad de empleado entre un engranaje de gobierno, el cual se refleja en el quehacer educativo en el aula, asimismo relacionando la mala calidad de la educación en la implementación de programas no surgidos de su contexto escolar.

Figura 2. La identidad de ser docente en el sistema educativo hondureño. 


\section{c. Estímulos}

\section{Unidad teórica}

Unidad empírica

Garcia ( 2008), "Muchos esfuerzos se han venido haciendo en países por mejorar la calidad del profesorado de nuestras escuelas. Ingentes cantidades de esfuerzos económicos y humanos se han puesto en el sistema con el fin de promover una enseñanza de mayor calidad. Y, sin embargo, siempre nos queda la duda de si todas esas actividades (fundamentalmente desarrolladas en torno a cursos de formación o capacitación) realmente tienen un impacto real en la enseñanza de los docentes y en los aprendizajes de sus alumnos. Tenemos la sensación de asistir a una especie de ceremonia que asume con facilidad y sin rubor que basta con que existan ocasiones en las que formalmente los profesores "son capacitados" para que el proceso de transferencia de aprendizaje en las aulas se produzca."

\section{Unidad de significado}

La categoría identificada es aumento al cumplir los cinco años de servicio, descartan que el sistema ofrezca recompensa por la labor educativa y además por título de profesionalización, versan que no existe un programa de incentivos.

Figura 3. Existencia de estímulos dentro del programa de inserción estímulos o recompensa económica en su desarrollo profesional. 


\section{d. Procedimientos de contratación}

\begin{tabular}{|c|c|}
\hline Unidad teórica & Unidad empírica \\
\hline $\begin{array}{l}\text { Avalos (2010), La estructura del sistema } \\
\text { escolar y su grado de convergencia con el } \\
\text { sistema de formación docente constituyen } \\
\text { también factores que inciden en las condi- } \\
\text { ciones de contratación y trabajo de los do- } \\
\text { centes noveles. }\end{array}$ & $\begin{array}{l}\text {...una tendencia sobre el último procedi- } \\
\text { miento al concurso de docente llevado en } \\
\text { el 2017; se constata, la forma controversial } \\
\text { de la "novedad de aplicar pruebas ", para la } \\
\text { inserción a la docencia y la misma "masiva } \\
\text { reprobación"... } \\
\text {... actualmente con el plan de transición } \\
\text { profesionalización docente muy bien, por- } \\
\text { que en recurso humano hay de excelencia... } \\
\text { He visto muchas deficiencias, según lo ob- } \\
\text { servado en la última convocatoria de plazas } \\
\text { para concurso. } \\
\text {... Es muy complicado, hasta cierto punto } \\
\text { comprendo que es necesario estar bien ca- } \\
\text { pacitado, pero le ponen muchos obstáculos } \\
\text { para poder entrar al sistema docente. }\end{array}$ \\
\hline \multicolumn{2}{|c|}{ Unidad de significado } \\
\hline \multicolumn{2}{|c|}{$\begin{array}{l}\text { Los procedimientos de contratación docente implementados actualmente, están con- } \\
\text { virtiéndose un factor determinante para el sistema educativo, una limitante y debilidad } \\
\text { de asegurar el perfil de un docente novel; sin prever la etapa de inserción un puente que } \\
\text { fomente la madurez y conocimiento del quehacer educativo en nuestro contexto hon- } \\
\text { dureño; esto articulado a un sistema nacional de formación docente, del cual se brinde } \\
\text { el seguimiento y evaluación al desarrollo profesional o continua, que posea un plan de } \\
\text { monitoreo de las capacitaciones que ostente habilidades, destrezas de entrada y salida. }\end{array}$} \\
\hline
\end{tabular}

Figura 4. Procedimientos de contratación para la inserción a la docencia. 


\section{Discusión}

Para argumentar las razones y utilidades que tendrá este estudio al mundo y específicamente a Honduras, representa un aporte significativo al conocimiento desde la contextualización de experiencias profesionales y académicas; por tanto, los argumentos aquí vertidos son parte de toda una revisión y análisis de documentos y sobre todo establecer valoraciones de experiencias en fenómenos educativos y sociales.

El papel fundamental que tiene la investigación científica, y para ello destacar los procedimientos técnicos que suelen generalizarse en el área de la investigación (los investigadores y las organizaciones que financian el objetivo global de la investigación para el desarrollo), el propósito debe incidir en el mejoramiento de la vida en las personas. Pero no se ve en sí, una política pública de "formación" como instrumento indispensable para transformar el conocimiento nuevo en una vida y un futuro mejor, debería ser la urgencia apremiante para la comunidad política.

La investigación científica es inversión para el desarrollo humano, convirtiéndose el hilo conductor para el cambio, y específicamente en ir gestando y perfilando la identidad de docente como agente facilitador de desarrollo humano sostenible. Es necesario, darse cuenta de las potencialidades que se lograría con el ingreso a la docencia en el marco de un "sistema integral"; es monumental -y a la vez fundamental - concretizar la iniciativa y lo reflejan los resultados de esta verificación empírica del estudio.

Para contextualizar esta idea de sistema en el caso de Honduras, actualmente está en vigencia la Ley Fundamental, orientada a lo prescrito en el marco de la nueva Ley General de Educación en Honduras. Sin duda es un buen inicio para el desarrollo de políticas favorecedoras para gestar un sistema de formación docente estratificado ${ }^{1}$, articulado con instituciones formadoras. Asimismo, orientado a destacar la importancia del papel que desarrollarían los programas de formación relacionados con la docencia, derivados de un "sistema", fundamentado ampliamente por su relevancia en el marco de una sociedad que demanda un verdadero cambio de modelo educativo en la educación. Este plantea un tipo de formación menos vinculada a un proceso de selección fragmentado y más centrado a capacidades, competencias, actitudes, destrezas, etc. Un factor determinante es el "tiempo", debido a que es una dimensión que se estructura en este enfoque como un asunto crucial; debe tener una administración con recepción racional, es decir en mecanismos profesionales e institucionales de responsabilidad sobre el uso del tiempo, en unas condiciones de flexibilidad que permitan aunar la autonomía y responsabilidad, las necesidades colectivas de un centro y cada uno de sus miembros.

1. Formación docente estratificado: Docentes noveles, intermedios, experimentado. 
Replantear con base a estas reflexiones es pensar que las iniciativas de poner en marcha modelos educativos para contribuir al desarrollo humano, definitivamente apuestan a inversiones en los sistemas educativos. Este planteamiento es la punta de lanza de la formación docente, convirtiendo a través de este, el rol del docente como agente de cambio desde un proceso de selección e inserción a la docencia, que abra las puertas al desarrollo integral del individuo, activa participación social y ciudadana. Además, dada la relación entre la educación y desarrollo humano para el bienestar común. ja; es decir, porque existe factores asociados a la formación, por ejemplo, la unidocencia constituye un porcentaje alto y no es atendida con metodología didáctica particular, como lo requiere. También estos factores generan las grandes brechas de desigualdad, jóvenes con rezago escolar, jóvenes con riesgo social relacionado a la inseguridad ciudadana, etc.

\section{Propuesta}

En el siguiente diagrama se muestra una propuesta de enfoque sistémico, basado en los resultados de la investigación:

Un sistema de formación docente en Honduras representa una problemática comple-

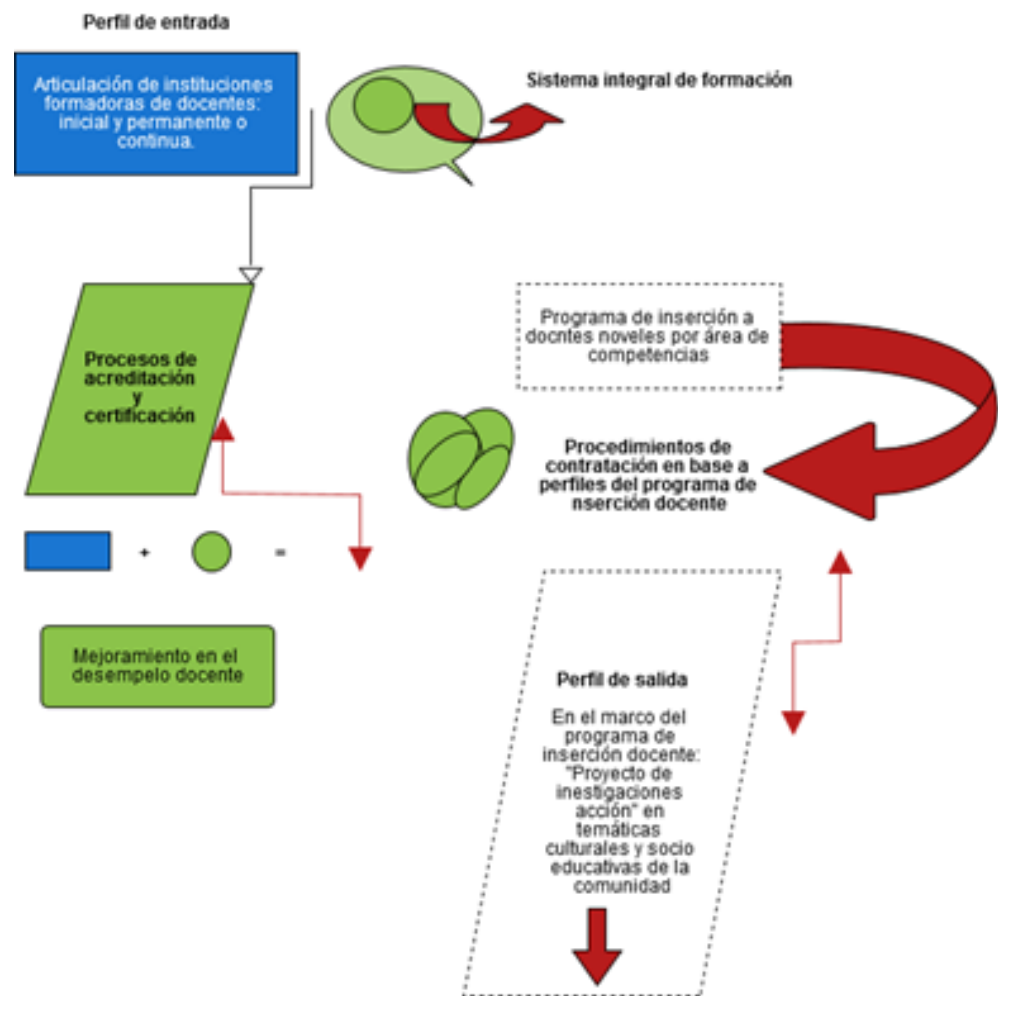

Figura 5. Enfoque sistémico al programa -inserción docente novel o principiante. 


\section{Referencias}

Asociación de Educación e Investigación en Ciencia de la Información de Iberoamérica y el Caribe (2007). Gestión de la información e investigación. Uruguay

Avalos, B. (2007). Formación docente continua y factores asociados a la política educativa en América Latina y el Caribe. Banco Interamericano de Desarrollo

Avalos, B.; Cavada, P.; Pardo, M. y Sotomayor, C. (2010). La profesión docente: temas y discusiones en la literatura internacional. Estudios pedagógicos, 36(1), 235-263

Carden, F. (2009). Del conocimiento a la política: del aprovechamiento de la investigación para el desarrollo. España: Centro Internacional de Investigaciones para el Desarrollo

Cornia, G. A. y Jolly, R. (1987). Ajuste con rostro humano. s.l.: Siglo XXI

Cuenca, R. y O'Hara, J. (2006). El estres en los maestros: percepción y realidad. Estudio de casos en Lima Metropolitana. Lima: Ministerio de Educación

Diario Oficial La Gaceta (22 de febrero de 2012). De la formación inicial docente y permanente (32).

Díaz Q., D. (2014). Competencias investigativas en procesos de formación docente: una experiencia de investigación acción a través de redes de docentes en servicio. Revista conocimiento educativo, $1,53-82$

Díaz Q., D. (s.f.). La unidocencia: desafios y retos con la apliacion del diseño curricular nacional básico (DCNB) en la formacion permanente en el marco del sistema nacional de formación docente. Revista Centroamericana de educación

Fonn, L. (2010). Estado de la educación nacional de Honduras. Tegucigalpa: UPNFM

Fundación para la Educación Ricardo Ernesto Maduro Andreu. (2017). Honduras: Informe de progreso educativo. Tegucigalpa

Galeano, M. (2004). Diseño de proyectos en la investigación cualitativa. Medellín: EAFITGarcía, C. (2009). El profesorado principiante: insercesión a la docencia. Barcelona: Octaedro

Griffin, K. (2001). Desarrollo humano: origen, evolución e impacto. En P. Ibara, y K. Unceta, Ensayos sobre el desarrollo humano (25-40). Barcelona: Icaria 
Hargreaves, A. (2003a). Profesorado, cultura y postmodernidad. Madrid: Morata

Hargreaves, A. (2003b). Profesorado, cultura y postmodernidad: cambian los tiempos, cambia el profesorado. s.l.: Ediciones Morata

Hernández, F. (2001). Bases metodológicas de la investigación educativa. Murcia: Diego Marín

Imbermón, F. (2007). La formación permanente del profesorado: nuevas ideas para formar en la innovacion y el cambio. España: Graó

Imbernón, F. (2009). La profesión docente ante los desafíos del presente y del futuro. s.l.: Universidad de Barcelona

Instituto de Investigaciones Científicas y Ecológicas (2009). Sistema Nacional de Formación Docente. Tegucigalpa

Manterola A., C. (1995). La formación docente, un reto imprescindible. Planiuc, 14(21)

Marcelo, C. (2011). Los comienzos en la docencia: un profesorado con buenos principios . Revista de currículum y formación del profesoradoMarchesi, A. (2007). Sobre el bienestar de los docentes: competencias, emociones y valores. s.l.: Alianza

Melgarejo D., J. (2005). El sistema educativo finlandés: la formacion del profesorado de educación primaria y secundaria obligatoria. s.l.: s.d.

Mezzadra, F. y Composto, C. (2008). Políticas para la docencia. Buenos Aires: Cippec

Millán P., A. (1982). Persona humana y justicia social. Madrid: Rialp

Ministerio de Educaciónde Chile (2005). Informe Comisión sobre Formación Inicial Docente

Moncada, G.; Alas S., M.; Orellana, D.; Hernández, R.; Chavez, M. y Hernández, B. D. (2003). Uso e impacto de la información empírica en la formulación y ejecución de política de educación básica en Honduras en el período 1990-2002. Tegucigalpa: PREAL/UPNFM

Moncada, G.; Alas S., M.; Orellana, D.; Hernández, R.; Chávez, M. y Hernández, B.D. (1999). Programa regional para la reforma educativa en América Latina. Teguciglpa: UPNFM

Muñecas V., A. (1995). Situación de la formación de docentes en América Latina. Planiuc, 14(21) 
Opazo, A. (2000). El sujeto de desarrollo humano. VIII Simposium de Eduación. Cátedra "Paulo Freire" "Educar para contruir el sueño: ética y conociiento en la transformacion soial". Discursos inaugurales y conferencias magistrales

Organización de las Naciones Unidas para la Educación, la Ciencia y la Cultura (1999). Declaración Mundial sobre Educación para todos. Jomtiem: Tailandia

Pacheco, F. (1996). Educación y sociedad en Costa Rica. Heredia: EFUNA

Peña, R. S. (2004). La formación inicial, profesionalización y capacitación docente en Honduras: transición hacia un nuevo sistema de formación docente. Copán

Pérez S., G. (1990). Investigación-acción: aplicaciones al campo social educativo. Madrid: Dykinson

Programa de las Naciones Unidas para el Desarrollo (2003). Informe sobre desarrollo humano: los objetivos de desarrollo del milenio: un pacto entre las naciones para eliminar la pobreza. New York: Mundi-Prensa

Programa de Promoción de la Reforma Eductiva de América Latina y el Caribe (2013). Situacion educativa de América Latina y el Caribe: hacia la educación de calidad para todos al 2015. Santiago: OREALC/UNESCO

Renaul, M. E. (2009). ¿Cómo se forman los docentes para las escuelas en Centroamérica? San José: CECC-SICA

Rodríguez, G. (1996). Metodologia de la investigacion cualitativa. Málaga, España: Aljibe

Ruiz, J. (2007). Metodología de la investigación cualitativa. Deusto: Universidad de Bilbao

Salgado P., R. (2004). La formacion inicial, profesionalizacion y capacitacion docente en Honduras: transición hacia un nuevo sistema de formación docente. Copán, Honduras

Salgado, R. U. (2010). Estado de la Educación Nacional. Tegucigalpa: UPNFM

Salgado, R. U. (2016). Desarrollo histórico de las instituciones formadoras de docentes y creacion de las escuelas normales: formación de maestros de primaria. Siglo XIX. 
Sandín, M. (2003). Investigación cualitativa en educación: fundamentos y tradiciones. Madrid: McGraw-Hill

Torres, R. (1995). La formación de los maestros: ¿qué se dice? ¿qué se hace? Santiago: CIDE/ UNESCO

Vaillant, D. (2007). Mejorando la formación y el desarrollo profesional docente en Latinoamerica. Revista pensamiento educativo, 41(2), 12

Vanegas, J.; Velez, C. y Vidarte, J. (2010). Aproximación a los fundamentos y métodos de investigación. Manizales: UAM

Weiis, C. H. (2009). Del conocimiento a la política: máximo aprovechamiento de la investigación para el desarrollo. Barcelona: Editorial Barcelona 